Review

\title{
MALAT1 as a Diagnostic and Therapeutic Target in Diabetes-Related Complications: A Promising Long-Noncoding RNA
}

Leila Elmi Abdulle ${ }^{1^{*}}$, Ji-long Hao ${ }^{1^{*}}$, Om Prakash Pant ${ }^{1^{*}}$, Xiu-fen Liu ${ }^{*}$, Dan-dan Zhou ${ }^{2^{*}}$, Ying Gao ${ }^{3^{*}}$, Abhishek Suwal $1^{*}$, Cheng-wei Lu ${ }^{1 凶}$

1. Department of Ophthalmology,

2. Department of Radiology,

3. Department of Endocrinology, The First Hospital of Jilin University, No. 71 of Xinmin St. Changchun, Jilin Province, 130021, China.

* Leila Elmi Abdulle, Ji-long Hao, Om Prakash Pant, Xiu-fen Liu, Dan-Dan Zhou, Ying Gao and Abhishek Suwal are co-first authors.

$\triangle$ Corresponding author: Cheng-wei Lu, M.D., Ph.D., Department of Ophthalmology, the First Hospital of Jilin University, No. 71 of Xinmin St. Changchun, Jilin Province, 130021, China. Email address: lcwchina800@sina.com; Telephone No: +8618684317115.

(C) Ivyspring International Publisher. This is an open access article distributed under the terms of the Creative Commons Attribution (CC BY-NC) license (https://creativecommons.org/licenses/by-nc/4.0/). See http://ivyspring.com/terms for full terms and conditions.

Received: 2018.09.20; Accepted: 2019.02.08; Published: 2019.04.20

\begin{abstract}
Diabetes mellitus is a global issue with increasing incidence rate worldwide. In an uncontrolled case, it can advance to various organ-related complications leading to an increase in morbidity and mortality. Long non-coding RNA (IncRNA) Metastasis-associated lung adenocarcinoma transcript 1 (MALAT1) appears to be a fairly novel IncRNA that is relevant to diabetes and its role in diabetic-related diseases initiation and progression have long been a subject of attention to many scholars. The expression of MALAT1 is elevated in different diabetic-related diseases. In this review, we demonstrate the various functions of MALATI in the different diabetes-related complications including ischemic reperfusion injury, retinopathy, cataract, atherosclerosis, cardiomyopathy, non-alcoholic steatohepatitis, gastroparesis, kidney disease, and gestational diabetes. The emerging evidence showed that the role of MALAT1 in diabetic-related complications is both pro-inflammatory and apoptosis in different cell types. These results concluded that MALAT1 is a potential diagnostic and future targeted therapy for diabetes-associated complications.
\end{abstract}

Key words: diabetes mellitus, diabetes-related complications, long non-coding RNA, MALAT1

\section{Introduction}

Diabetes mellitus (DM) is a clinical condition characterized by a high glucose level either due to decreased insulin level or due to insulin insensitivity [1]. The International Diabetes Federation (IDF) estimated on the overall prevalence of DM to be 366 million in 2011, and it is predicted to rise to 552 million by 2030 [2]. In uncontrolled cases, DM can affect various organs including the brain, eye, heart, stomach, kidney and liver leading to various severe and organ-threatening complications [3-5]. Currently, there is no specific biomarker for diabetes complicated diseases as well as treatment regimens that could halt and prevent the disease progression. Hence it is urgent to explore the specific biomarkers to diagnose, detect, and catch the early sequence of disease development.
Long non-coding RNA (lncRNA) are welldefined enormous and different class of transcribed RNA molecules with dimension of more than 200 nucleotides but lack an open reading structure of considerable size. Similar to the protein-coding RNAs, most of the IncRNA are RNA polymerase II transcripts with a 5 'cap and poly-A tail. The majority of lncRNA are predominant inside the cell nucleus and show either low revolutionary conservation or lesser expression level than mRNAs [6, 7]. LncRNA is an important regulator of various biological processes, including proliferation, differentiation, invasion and apoptosis [8]. Hence, lncRNA are evolving as new biomarkers and therapeutic targets in several human diseases [9].

LncRNA-Metastasis-associated lung adenocarc- 
inoma transcript 1 (MALAT1) is among well studied and highly conserved lncRNA, which was linked to a variety of pathological processes including diabetes-related complications and various malignancies [10]. MALAT1 coding gene is located on the short arm of human chromosome 11q13.1, and its transcript is approximately $8 \mathrm{~kb}$ [11].

Recent studies had revealed that MALAT1 can play significant roles in the pathophysiological process, tissue inflammation, tumor progression, angiogenesis, cardiovascular remodelling, liver fibrosis, and diabetes progression by modulating gene transcription [12]. In this review, we focused on the correlation between MALAT1 and diabetes-related complications and highlighted the currently advanced research evolutions on the different expression pattern of MALAT1, along with the precise biological role in those diseases (Table 1). MALAT1 may act as an innovative biomarker and therapeutic target for the diagnosis and treatment of diabetes-related complications and disease prognosis assessment. Furthermore, MALAT1- targeting therapeutic inventions may be identified as a promising prevention and treatment option for associated diabetic diseases.

\section{MALAT1 in Diabetes-Related Complications}

\section{Cerebral Ischemic Reperfusion Injury}

Cerebrovascular disease has a high incidence of neurological disorders, disability and mortality that severely depreciate the human health [13]. DM is considered as one of the key independent risk factors for the development and exacerbation of ischemic reperfusion injury. DM can induce cerebrovascular diseases, which lead to cranial nerve injuries or die [14-16].

MALAT1 mediates the production of glucoseinduced inflammatory cytokines, namely tumor necrosis factor alpha (TNF-a) and interleukin 6 (IL-6), in the endothelial cells which promote DM- associated vascular insult [17]. MALAT1, MyD88, IRAK1 and TRAF6 were notably up-regulated by DM-ischemic reperfusion models contrasted to the ischemic reperfusion models both in vivo and in vitro. Subsequently, MyD88-dependent signalling provoked cytokine activation and inflammatory reactions for the development of cerebral ischemic reperfusion injury [18]. MALAT1 up-regulation increased MyD88 adaptor proteins and TRAF6 as well as IRAK1 and again get into a complex, resulting in the activation of NF-kB cascade reaction [19]. On an overall, the pre-cortical infracted tissues from ischemic reperfusion injury model in diabetic rats showed a severe form of neuronal damage by presenting significant neurological deficit scores and increasingly brain oedema. MALAT1 was therefore as a component for DM-associated cerebral ischemic reperfusion injury via positive regulation of MyD88 expression as well as increasing H3 histone acetylation of the MyD88 promoter [20].

Therefore, MALAT1 is obviously an essential regulatory factor for ischemic reperfusion injury due to DM. It could be an effective therapeutic target for the management and future prevention of ischemic reperfusion injury-induced by DM. However, more in-depth studies are needed to give more insights on MALAT1 MyD88 promoter acetylation mechanism.

\section{Diabetic Retinopathy}

Diabetic retinopathy (DR) is a devastating ocular impediment and the leading cause of vision loss among working-age adults in developed countries [21]. Worldwide, the prevalence of DR has been predicted to be $35 \%$ and the prevalence of vision-impairing is estimated around $10 \%[22,23]$. Various risk factors such as poor glycaemic control, hypertension, hyperlipidaemia, longer diabetes duration and albuminuria are reported to be the origination and development of DR [24, 25].

Table 1. MALAT1 Influences in Diabetes-associated Complications

\begin{tabular}{|c|c|c|c|c|c|}
\hline Diseases & Expression & Related molecules & Model & Role & References \\
\hline Cerebrovascular Disease & Up-regulated & MyD88, IRAK1, TRAF6 & In vitro and in vivo & Inflammation & 20 \\
\hline Diabetic Retinopathy & Up-regulated & PRC2, IL-6 and TNF-a & In vivo and vitro & Proliferation & 27,26 \\
\hline Diabetic Cataract & Up-regulated & p38,SP1 & In vivo and vitro & Apoptosis & 37 \\
\hline Atherosclerosis & Up-regulated & NLRP3, ELAVL1, miR-23c & In vivo & Apoptosis & 41 \\
\hline Diabetic Cardiomyopathy & Up-regulated & TNF-a, IL-1 $\beta$, IL-6, NO & In vitro and in vivo & Apoptosis & 45,46 \\
\hline Non-alcoholic Steatohepatitis & Up-regulated & CXCL5 & In vivo & Inflammation & 53 \\
\hline Diabetic Gastroparesis & Up-regulated & a-SMA, SM- myosin & In vivo & - & 61,72 \\
\hline Diabetic Kidney Disease & Up-regulated & $\begin{array}{l}\text { SAA3, IL-6, ELAVL1, NLRP3, } \\
\text { Caspase-1, SRSF1 }\end{array}$ & In vitro and in vivo & Inflammation, pyroptosis & $65-67$ \\
\hline Gestational Diabetes & Up-regulated & - & In vivo & - & 71 \\
\hline
\end{tabular}

Note: MyD88: myeloid differentiation factor-88 adaptor protein; IRAK1: interleukin-1 receptor-associated kinase; TRAF6: tumor necrosis factor receptor-associated factor 6; PRC2: polycomb repressive complex 2; IL-6: interleukin-6; TNF-a: tumor necrosis factor-a; SP1: specificity protein 1; NLRP3: NOD-like receptor family, pyrin domain containing 3; ELAVL1: embryonic lethal, abnormal vision-like; IL-1 $\beta$ : interleukin-1 $\beta$; IL-6: interleukin-6; NO: nitric oxide; CXCL5: C-X-C motif chemokine ligand 5; a-SMA: alpha smooth muscle actin; SM myosin: smooth muscle myosin; SAA3: serum amyloid antigen 3; SRSF1: serine/arginine splicing factor. 
Biao et al. reported that MALAT1 is considerably up-regulated in the diabetic mice retinas, RF/6A cell model of hyperglycaemia, aqueous humour samples, and fibrovascular membranes (FVMs) of diabetic patients. Thus, they documented that MALAT1 dysregulation might contribute to the occurrence of DR via the in vitro study, experimental animal study and the analysis of clinical samples [26]. MALAT1 is also capable of implicating the expressions of inflammatory transcripts through association with the components of the PRC2 complex in diabetes. Likewise, vitreous humour collected from diabetic patients has shown expressions of MALAT1, IL-6, and TNF-a. Unusually, the DNA methylation array revealed that transient high glucose contact in human retinal microvascular endothelium cells (HRECs) does not add to significant methylation modifications at CpG sites through the MALAT1 gene. Conversely, overall inhibition of DNA methyltransferases prompted the major increase in MALAT1 and related inflammatory transcripts in HRECs [27].

Guo et al. also explained that methyl donor could diminish MALAT1 expression in this cellsentailing that DNA methylation involved the regulation of MALAT1 expression [28]. As a result, it is not a confounding that MALAT1 dysregulation may perhaps disrupt the proliferation and migration of FVM-related cells, which affect the DR pathogenesis. In another study, MALAT 1 expression was highly up-regulated in STZ-induced diabetic rat's retina and $\mathrm{db} / \mathrm{db}$ mice. MALAT1 ablation enhances DR in vivo. Furthermore, MALAT1 knockdown regulates migration and retinal endothelial cell proliferation. MALAT1 over-expression denotes a crucial pathogenic mechanism for diabetes-related microvascular dysfunction, hyperproliferation of endothelial cells through p38MAPK signalling. MALAT1 inhibition may become a potent anti-angiogenic therapy for diabetic microvascular complications [29]. All these findings collectively determine the impact of MALAT1 in inflammation and epigenetic regulation in DR.

In conclusion, MALAT1 a preserved lncRNA may turn out to be a potential biomarker for the diagnosis and prognosis of DR. More studies are essential to examine the correlations among lncRNA change and DR development at different stages. Additionally, in vivo and in vitro studies should be conducted to clarify the molecular mechanisms of lncRNA mediated DR occurrence and evaluate their potential for the diagnosis, prognosis and treatment of DR.

\section{Diabetic Cataract}

Diabetic cataract (DC) commonly occurs earlier and develops rapidly [30, 31]. Regardless of the successful implantation with synthetic intraocular lenses, cataract remains one of the leading causes of blindness [32, 33]. Although there are no treatment guidelines for DC, there is a need to understand the progression and pathogenesis of cataract formation to deliver therapeutic targets for the prevention and treatment of DC apart from surgical methods.

A recent study revealed that the expression of MALAT1 was elevated in diabetic cataract tissues cells, hyperglycaemia (HG)-induced human lens epithelial cells (HLECs) as well as up-regulated by HG to provoke the apoptosis and oxidative stress of HLECs. Moreover, HG prompted the up-regulation of MALAT1 by SP1 binding of MALAT1 promoter regions to exert its role in the apoptosis oxidative stress of HLECs [12].

Yuan et al. further studied the mechanism between MALAT1 and the apoptosis as well as oxidative stress of HLECs and discovered that p38MAPK was up-regulated in HG-treated HLECs. Likewise, previous studies demonstrated that p38MAPK play a pivotal role in HLEC activity and apoptosis induced by oxidative stress [34-36]. Meanwhile, knockdown of p38MAPK restrained the influence of MALAT1 over-expression on HLECs [37].

Hence, it was confirmed that MALAT1 promote the apoptosis and oxidative stress of HLECs through the initiation of the p38MAPK signalling pathway [37]. Thus, MALAT1 might be a likely therapeutic target for DC.

\section{Atherosclerosis}

Hyperglycemia is recognized as an independent cardiovascular risk factor. It can accelerate and heightened atherosclerosis, which is a principal reason for morbidity as well as mortality in DM [38]. The incidence of coronary heart disease and peripheral vascular disease increases to 4 times and more 10 times in a patient with DM as compared to the normal individual [39]. Therefore, it begs to discover the precise molecular processes involved in the development as well as acceleration of atherosclerosis in DM patient and to accomplish possible therapeutic targets.

Recent evidence revealed that inflammation coupled with over-activated innate immunity is strongly associated with the pathogenesis of DM and related complications comprising atherosclerosis [40]. It was revealed that MALAT1 plays an important role in the development and acceleration of diabetic atherosclerosis. Han et al. found that MALAT1 was highly expressed in the macrophages of diabetic atherosclerosis rats along with hypernomic stimulation of NLRP3 inflammasome though sponging 
miR-23c as well as an increase in the building of systemic inflammatory cytokines. They also suggested that the administration of low-dose sinapic acid (SA) suppresses MALAT1-mediated NLRP3 inflammasome activation, pyroptosis of macrophages as well as generalized inflammation [41].

To sum up, MALAT1 might act as an inflammatory factor in the development of diabetic atherosclerosis. The interaction between MALAT1 and low-dose SA may provide a new therapeutic direction in diabetic atherosclerosis.

\section{Diabetic Cardiomyopathy}

Diabetic cardiomyopathy (DC) is an essential cardiovascular system (CVS) complication of DM characterized by a variability of morphological changes, comprising myocyte hypertrophy, myofibril depletion, interstitial fibrosis, as well as intramyocardial microangiopathy [42]. It arises autonomously from hypertension, dyslipidemia as well as coronary artery disease and bears a considerable risk for the sequential development of clinical heart failure along with higher morbidity as well as mortality [43]. High blood sugar level is a main etiological factor responsible in the occurrence of DC and upsurges the risk of heart failure by numerous folds [44]. CVS diseases are responsible for the utmost mortality rate. Hence, it is evident that initial prevention, as well as the development of cardiac function, would significantly decrease the prevalence.

Zhang et al. suggested that MALAT1 was considerably upregulated in DM rats. In addition, TNF-a, IL-1 $\beta$ along with IL-6 levels were uncharacteristically high in the diabetic myocardium. MALAT1 knockdown could appreciably decrease inflammatory cytokine concentration, signifying that MALAT1 might be involved in the development of DCM [45]. Furthermore, MALAT1 knockdown could significantly decrease cardiomyocyte apoptosis in the DM positive MALAT1-shRNA group [46].

In another study, Bacci et al. concluded that decrease of nitric oxide (NO) or cGMP bio-availability produced by long-standing DM impairs lncRNA expression as well as sildenafil and reestablishing function of NO signaling normalized MALAT1 expression levels, reducing the cardiac symptoms [47]. In conclusion, MALAT1 is considerably overexpressed in cardiac tissue of DM rats, and its inhibition results in improvement in the cardiac function. Thus, knockdown of MALAT1 may serve as a novel therapeutic approach for DC.

\section{Non-alcoholic Steatohepatitis}

Non-alcoholic steatohepatitis (NASH) is recognized as the major cause of chronic liver disease as well as an important cause of cryptogenic cirrhosis [48]. NASH patients experience higher liver associated morbidity along with mortality [49, 50]. Additionally, it has been anticipated to be the foremost reason for liver transplantation within the following few years [51].

A recent study emphasized that MALAT1, well-known for involvement in the progression of liver carcinoma may also play a key role in the occurrence of NASH as well as fibrosis in patients with Non-Alcoholic fatty liver disease (NAFLD) via a chemokine-mediated process [52]. Several lncRNA including nuclear paraspeckle assembly transcript 1 , hepatocellular carcinoma upregulated lncRNA, and MALAT1 were tremendously expressed in liver fibrosis comparative to normal tissue. Moreover, the potential target of MALAT1 has been identified as C-X-C motif chemokine ligand 5 (CXCL5). However, CXCL5 showed discrepancy expression among different histologic cell types. Knockdown of MALAT1 by siRNA diminished protein levels and CXCL5 transcript by $30 \%$ and $50 \%$ respectively in HepG2 cells, indicating that MALAT1 plays a role in CXCL5 expression regulation [53].

Notable, hyperglycaemia control MALAT1 expression and treatment of high glucose with the hepatic cells resulted in increased levels of MALAT expression over time in HepG2 cells but no change in LX-2 cells [53]. High glucose, as well as type 2 diabetes, are associated with NAFLD severity along with development of hepatic inflammation and fibrosis [54-56].

These conclusions uphold a role for CXCL5 in the pathogenesis of liver disease, possibly through a MALAT1-mediated mechanism. Further studies will be required on the association between MALAT1 and CXCL5.

\section{Diabetic Gastropathy}

Gastroparesis is a symptomatic disorder of the stomach defined by delayed emptying of ingested food particles. DM along with idiopathic factors contributes nearly $60 \%$ of gastroparesis cases [57]. Previous studies demonstrated that gastrointestinal symptoms occur in about $75 \%$ of the diabetic cases, while approximately $30-50 \%$ of these cases were due to diabetic gastropathy [58-60]. However, the prevalence of gastroparesis in diabetes mellitus type 1 (T1DM) varies considerably. In addition, $40 \%$ of T1DM patients were diagnosed with gastroparesis in tertiary centres [60].

Recently, IncRNA MALAT1 was developed as a probable regulator in the pathogenesis of DGP. Smooth muscle cells (SMC) contractility marker proteins were found to be decreased in gastric models 
of DM mouse and MALAT1 expression was higher in DGP rat model. Secondly, MALAT1 was overexpressed in the adjacent health tissues taken from diabetic gastric cancer patients with DGP. In human gastrointestinal SMCs, high sugar levels augmented the MALAT1 expression. MALAT ablation shortened the cell viability, suppressed impending of the cell migration and carried cell death in human gastric SMCs preserved with high glucose. And, MALAT1 suppression induced a-SMA and SM myosin heavy chains expression, prompting the SMCs to express contractility markers [61].

Hence, MALAT1 was associated with the progression of DGP leading to phenotype alteration and influencing normal cellular processes of smooth muscle cells. However, more intensive laboratories works are necessary to the highlight the precise mechanism and function of lncRNA MALAT1 in SMC phenotype switch.

\section{Diabetic Kidney Disease}

Diabetic kidney disease is an advancing condition that progresses secondary to DM [62]. It is the principal cause of chronic renal failure accounting for nearly $50 \%$ of all end-stage renal disease [63, 64]. Existing treatment modalities are directed towards the decreasing blood pressure as well as blood sugar to reduce albuminuria and slow disease progression. However, in the majority of cases, there are no reliable methods to prevent the development of diabetic kidney disease and chronic renal failure. Thus, there is an urgent need for therapeutic targets for drug development as well as diagnostic markers for clinical treatments to prevent the development and progression of diabetic kidney disease.

MALAT1 was notably highly expressed in kidney tissues from C57BL/6 mice with streptozocininduced diabetic kidney disease. $\mathrm{Hu}$ et al. reported that early interfering with MALAT1 siRNA partly re-established podocytes function as well as proscribed b-catenin nuclear accumulation and SRSF1 overexpression. Furthermore, b-catenin was involved in MALAT1 transcription by binding to the promotor region of MALAT1; b-catenin knock-down also decreased MALAT1 levels [65]. In addition, Li et al. reported that with an increase in MALAT1 expression there was a decrease in miR-23c in streptozotocininduced diabetic rats as well as in high-glucosetreated HK-2 cells. Down-regulation of MALAT1 inhibited the expression of ELAVL1, NLRP3, Caspase- 1 as well as the pro-inflammatory cytokine IL-1 $\beta$ and up-regulated the expression of miR-23c. Besides, luciferase assays revealed that the expression of MALAT1 antagonized the effect of miR-23c on the down-regulation of its target ELAVL1 and inhibited hyperglycemia-induced cell pyroptosis [66].

In another study, Wu et al., showed that expression level of MALAT1, as well as its downstream target SAA3, was considerably down-regulated in renal tissues after bariatric surgery in rats, which in turn reduced the expression of the pro-inflammatory cytokines IL-6 and TNF-a. Knockdown of MALAT1 in HK-2 cell lines further confirmed that expression levels of SAA3, IL-6, and TNF-a were regulated by MALAT1 under both lowand high-glucose conditions [67].

Therefore, MALAT1 plays a key pivotal role in diabetic nephropathy and recognizing this mechanism may ultimately contribute to the progress of novel lncRNA-based therapeutic strategies for the management of diabetic kidney disease.

\section{Gestational Diabetes Mellitus}

Gestational diabetes mellitus (GDM) is a type of diabetes characterized by glucose intolerance with onset during pregnancy, resulting in high blood sugar level with varying severity [68]. Recent studies had shown that one in seven births were affected by this disease, regardless of the reduced global number of cases which has fallen to 21.0 million [68]. Likewise, other type of hyperglycaemias, GDM also redirects a functional difference between a person's insulin secretion and insulin demand [69]. GDM prevalence is excessive in summer, perhaps reflecting an association between blood glucose levels and temperature. The exact mechanism by which temperature may influence glucose metabolism in pregnancy remains uncertain [70]. Although, lncRNA and DM have attracted the attention of many scholars, but only limited research have focused on GDM.

Zhang et al. investigated the relationship between lncRNA MALAT1, lncRNA p21, IncRNA H19 and GDM. They reported that the expression level of lncRNA MALAT1 was considerably higher in the GDM group as compared to the non-GDM group. Furthermore, IncRNA MALAT1 correlated with the expression of lncRNA p21 and lncRNA H19 [71].

MALAT1 was identified as a novel serum biomarker to predict GDM. This provides a promising biomarker for future strategy to diagnose and treat GDM by regulating the expression of lncRNA MALAT1. However, in-depth studies are needed.

\section{Conclusion and Future Perspectives}

Increasing evidence discovered that lncRNA plays a vital role in the pathogenesis and progression of diabetes-related complicated diseases including ischemic reperfusion injury, retinopathy, cataract, atherosclerosis, cardiomyopathy, non-alcoholic steatohepatitis, gastroparesis, kidney disease and 
gestational diabetes mellitus. The clinical significance of MALAT1 and its molecular mechanisms in controlling these diseases are explained. This review clarifies the sophisticated researches and progresses with the possible roles of MALAT1 in different diabetic-related diseases (Figure 1). Studies had shown that the expression pattern and role of MALAT1 was similar in different types of diseases, and was up-regulated (Table 1). The expression trend of MALAT1 was consistent and the role of MALAT was even matching in the same type of cell in different researches. Additionally, MALAT1 associated number of dysregulated diseases and elevated MALAT1 levels could be a number of reasons for instance, gastric cancer, and hepatocellular carcinoma; thus, to determine the specificity of MALAT1 in the clinic, other investigations should be carried out to rule out if there are no other underlying causes. Overall, increased expression levels of MALAT1 in different diabetes-related complications, as well as MALAT1 therapeutic targeting by synthetic oligonucleotides, including miRNAs and siRNAs, have established MALAT1 as a both therapeutic target and potential biomarker. Nevertheless, there is still lack of the independent cohort study to validate, and additional miRNAs interplay and epigenetic modifications are needed, to advance the development of better-targetted therapeutic strategies. Hence, multicentre studies will be vital, which can add to the clinical utility of MALAT1 as an effective biomarker.
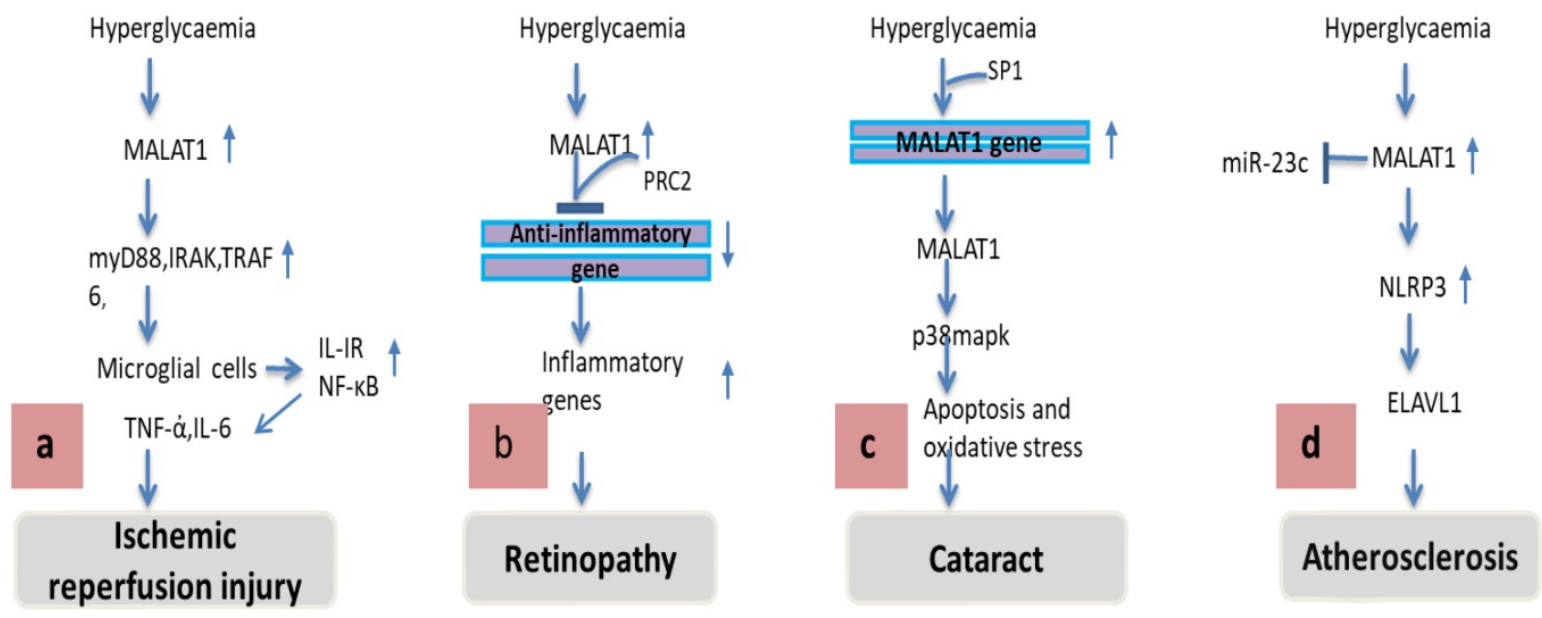

\section{Hyperglycaemia $\rightarrow$ MALAT1}
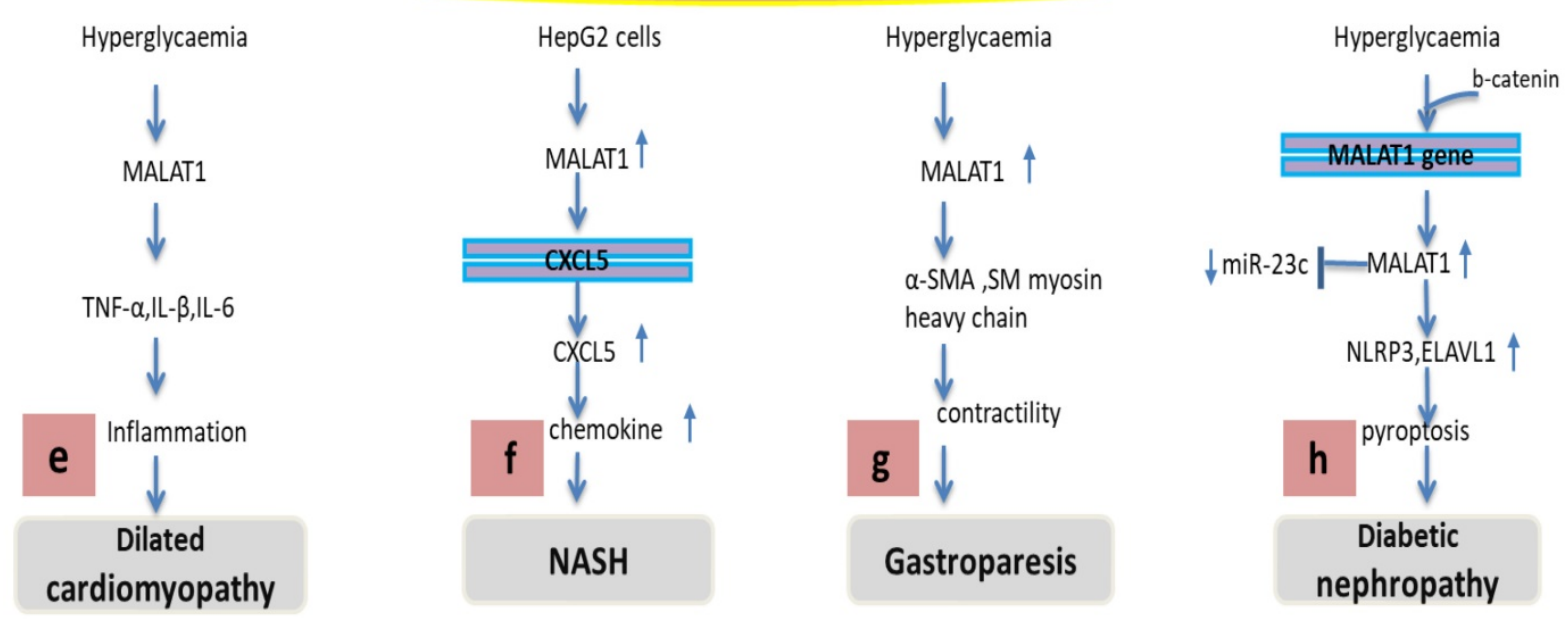

Figure 1. Various regulatory mechanisms of MALAT1 in different diabetes-related complications. (A) Hyperglycaemia-induced MALAT1 trigger an inflammatory response in microglial cells via activation of MyD88 signalling leading to ischemic reperfusion injury. (B) MALAT1 recruits PRC2 to bind anti-inflammatory gene promoter which suppresses its transcription and subsequently increases inflammatory response leading to retinopathy. (C) Hyperglycaemia induces Spl protein binding to the MALATI promoter gene increasing MALATI expression, increased MALATI activates P38MAPK pathway causing apoptosis and oxidative stress leading to cataract formation. (D) MALAT1 inhibits miR-23 which in turn increases ELAVL1 and NLRP3 leading to atherosclerosis. (E) Hyperglycaemia promotes MALAT1 levels which trigger inflammatory cytokines TNF- $\alpha$, IL-1 $\beta$ and IL-6 leading to inflammation and cardiomyopathy. (F) MALAT1 promotes $\mathrm{cxcl5}$ chemokine via $\mathrm{cxcl5}$ gene transcript which triggers inflammation, fibrosis and NASH. (G) MALATI decreases $\alpha$-SMA, SM myosin heavy chain diminishes contractility leading to gastroparesis. (H) MALATI antagonized the effect of miR-23 on its target ELAVLI, NLRP3 promoting pyroptosis and diabetic nephropathy. 


\section{Abbreviations}

LncRNA: Long non-coding RNA; MALAT1: Metastasis-associated lung adenocarcinoma transcript 1; DM: Diabetes mellitus; MyD88: myeloid differentiation factor-88 adaptor protein; IRAK1: interleukin-1 receptor-associated kinase; TRAF6: tumor necrosis factor receptor-associated factor 6; DR: Diabetic retinopathy; PRC2: polycomb repressive complex 2; IL-6: interleukin-6; TNF-a: tumor necrosis factor-a; DC: Diabetic cataract; SP1: specificity protein 1; NLRP3: NOD-like receptor family pyrin domain containing 3; ELAVL1: embryonic lethal abnormal vision-like 1; DC: Diabetic cardiomyopathy; TNF-a: tumor necrosis factor- $\alpha$; IL-1 $\beta$ : interleukin-1 $\beta$; IL-6: interleukin-6; NO: nitric oxide; NASH: Non-alcoholic steatohepatitis; CXCL5: C-X-C motif chemokine ligand 5; a-SMA: alpha-smooth muscle actin; SM-myosin: smooth muscle myosin; SAA3: serum amyloid antigen 3; SRSF1: serine/arginine splicing factor; GDM: Gestational diabetes mellitus.

\section{Acknowledgments}

We appreciate the support from the grants by the National Natural Science Foundation of China (Grant No. 81800828).

\section{Competing Interests}

The authors have declared that no competing interest exists.

\section{References}

1. Guay C, Regazzi R. Circulating MicroRNAs as Novel Biomarkers for Diabetes Mellitus. Nat Rev Endocrinol. 2013; 9: 513-21.

2. Whiting DR, Guariguata L, Weil C, Shaw J. IDF Diabetes Atlas: Global Estimates of the Prevalence of Diabetes for 2011 and 2030. Diabetes Res Clin Pract. 2011; 94: 311-21.

3. Hanefeld M, Duetting E, Bramlage P. Cardiac Implications of Hypoglycaemia in Patients with Diabetes - A Systematic Review. Cardiovasc Diabetol. 2013; 12: 135 .

4. Jenkins AJ, Joglekar MV, Hardikar AA, Keech AC, O'Neal DN, Januszewski AS. Biomarkers in Diabetic Retinopathy. Rev Diabet Stud. 2015; 12: 159-95.

5. Deshpande AD, Harris-Hayes M, Schootman M. Epidemiology of Diabetes and Diabetes-Related Complications. Phys Ther. 2008; 88: 1254-64.

6. Rinn JL, Chang HY. Genome regulation by long noncoding RNAs. Annu Rev Biochem. 2012; 81: 145-66.

7. Ravasi T, Suzuki H, Pang KC, Katayama S, Furuno M, Okunishi R, et al. Experimental validation of the regulated expression of large numbers of non-coding RNAs from the mouse genome. Genome Res. 2006; 16: 11-9.

8. Liu XF, Hao JL, Xie T, Pant OP, Lu CB, Lu CW, et al. The BRAF activated non-coding RNA: A pivotal long non-coding RNA in human malignancies. Cell Prolif. 2018; 51: e12449.

9. Weidle UH, Birzele F, Kollmorgen G, Ruger R. Long Non-coding RNAs and their Role in Metastasis. Cancer Genomics Proteomics. 2017; 14: 143-60.

10. Su Y, Wu H, Pavlosky A, Zou LL, Deng X, Zhang ZX, et al. Regulatory non-coding RNA: new instruments in the orchestration of cell death. Cell Death Dis. 2016; 7: e2333.

11. Wilusz JE, Freier SM, Spector DL. 3' end processing of a long nuclear-retained noncoding RNA yields a tRNA-like cytoplasmic RNA. Cell. 2008; 135: 919-32.

12. Lei L, Chen J, Huang J, Lu J, Pei S, Ding S, et al. Functions and regulatory mechanisms of metastasis-associated lung adenocarcinoma transcript 1 . J Cell Physiol. 2018 ; 234: 134-151.

13. Backhouse EV, McHutchison CA, Cvoro V, Shenkin SD, Wardlaw JM. Early life risk factors for cerebrovascular disease: A systematic review and meta-analysis. Neurology. 2017; 88: 976-84.

14. Lincoff AM, Nicholls SJ, Riesmeyer JS, Barter PJ, Brewer HB, Fox KAA, et al. Evacetrapib and Cardiovascular Outcomes in High-Risk Vascular Disease. N Engl J Med. 2017; 376: 1933-42.
15. Paneni F, Luscher TF. Cardiovascular Protection in the Treatment of Type 2 Diabetes: A Review of Clinical Trial Results Across Drug Classes. Am J Cardiol. 2017; 120: S17-S27.

16. Hamed SA. Brain injury with diabetes mellitus: evidence, mechanisms and treatment implications. Expert Rev Clin Pharmacol. 2017; 10: 409-28.

17. Puthanveetil P, Chen S, Feng B, Gautam A, Chakrabarti S. Long non-coding RNA MALAT1 regulates hyperglycaemia induced inflammatory process in the endothelial cells. J Cell Mol Med. 2015; 19: 1418-25.

18. Gao Y, Fang X, Tong Y, Liu Y, Zhang B. TLR4-mediated MyD88-dependent signaling pathway is activated by cerebral ischemia-reperfusion in cortex in mice. Biomed Pharmacother. 2009; 63: 442-50.

19. Nishida A, Inatomi $O$, Fujimoto $T$, Imaeda $H$, Tani $M$, Andoh $A$. Interleukin-36alpha Induces Inflammatory Mediators From Human Pancreatic Myofibroblasts Via a MyD88 Dependent Pathway. Pancreas. 2017; 46: 539-48.

20. Wang LQ, Zhou HJ. LncRNA MALAT1 promotes high glucose-induced inflammatory response of microglial cells via provoking MyD88/IRAK1/TRAF6 signaling. Sci Rep. 2018; 8: 8346.

21. Klein BE. Overview of epidemiologic studies of diabetic retinopathy. Ophthalmic Epidemiol. 2007; 14: 179-83

22. Leasher JL, Bourne RR, Flaxman SR, Jonas JB, Keeffe J, Naidoo N, et al. Erratum. Global Estimates on the Number of People Blind or Visually Impaired by Diabetic Retinopathy: A Meta-analysis From 1990-2010. Diabetes Care 2016;39:1643-1649. Diabetes Care. 2016; 39: 2096.

23. Rajavi Z, Safi S, Javadi MA, Azarmina M, Moradian S, Entezari M, et al. Diabetic Retinopathy Clinical Practice Guidelines: Customized for Iranian Population. J Ophthalmic Vis Res. 2016; 11: 394-414

24. Fu YP, Hallman DM, Gonzalez VH, Klein BE, Klein R, Hayes MG, et al. Identification of Diabetic Retinopathy Genes through a Genome-Wide Association Study among Mexican-Americans from Starr County, Texas. J Ophthalmol. 2010; 2010.

25. Doria A. Genetics of diabetes complications. Curr Diab Rep. 2010; 10: 467-75.

26. Yan B, Tao ZF, Li XM, Zhang H, Yao J, Jiang Q. Aberrant expression of long noncoding RNAs in early diabetic retinopathy. Invest Ophthalmol Vis Sci. 2014; 55: 941-51.

27. Biswas S, Thomas AA, Chen S, Aref-Eshghi E, Feng B, Gonder I, et al. MALAT1: An Epigenetic Regulator of Inflammation in Diabetic Retinopathy. Sci Rep. 2018; 8: 6526.

28. Guo F, Guo L, Li Y, Zhou Q, Li Z. MALAT1 is an oncogenic long non-coding RNA associated with tumor invasion in non-small cell lung cancer regulated by DNA methylation. Int J Clin Exp Pathol. 2015; 8: 15903-10.

29. Liu JY, Yao J, Li XM, Song YC, Wang XQ, Li YJ, et al. Pathogenic role of IncRNA-MALAT1 in endothelial cell dysfunction in diabetes mellitus. Cell Death Dis. 2014; 5: e1506.

30. Harding JJ, Egerton M, van Heyningen R, Harding RS. Diabetes, glaucoma, sex, and cataract: analysis of combined data from two case control studies. Br J Ophthalmol. 1993; 77: 2-6.

31. Kahn HA, Leibowitz HM, Ganley JP, Kini MM, Colton T, Nickerson RS, et al. The Framingham Eye Study. II. Association of ophthalmic pathology with single variables previously measured in the Framingham Heart Study. Am J Epidemiol. 1977; 106: 33-41.

32. Pollreisz A, Schmidt-Erfurth U. Diabetic cataract-pathogenesis, epidemiology and treatment. J Ophthalmol. 2010; 2010: 608751.

33. Pascolini D, Mariotti SP. Global estimates of visual impairment: 2010. Br J Ophthalmol. 2012; 96: 614-8.

34. Yuan L, Wang J, Xiao H, Wu W, Wang Y, Liu X. MAPK signaling pathways regulate mitochondrial-mediated apoptosis induced by isoorientin in human hepatoblastoma cancer cells. Food Chem Toxicol. 2013; 53: 62-8.

35. Wu WC, Hu DN, Gao HX, Chen M, Wang D, Rosen R, et al. Subtoxic levels hydrogen peroxide-induced production of interleukin- 6 by retinal pigment epithelial cells. Mol Vis. 2010; 16: 1864-73.

36. Law AH, Tam AH, Lee DC, Lau AS. A role for protein phosphatase $2 \mathrm{~A}$ in regulating p38 mitogen activated protein kinase activation and tumor necrosis factor-alpha expression during influenza virus infection. Int J Mol Sci. 2013; 14: 7327-40.

37. Gong W, Zhu G, Li J, Yang X. TEMPORARY REMOVAL: "LncRNA MALAT1 promotes the apoptosis and oxidative stress of human lens epithelial cells via p38MAPK pathway in diabetic cataract". Diabetes Res Clin Pract. 2018.

38. Shrikhande GV, Scali ST, da Silva CG, Damrauer SM, Csizmadia E, Putheti P, et al. O-glycosylation regulates ubiquitination and degradation of the anti-inflammatory protein A20 to accelerate atherosclerosis in diabetic ApoE-null mice. PLoS One. 2010; 5: e14240.

39. Faxon DP, Fuster V, Libby P, Beckman JA, Hiatt WR, Thompson RW, et al. Atherosclerotic Vascular Disease Conference: Writing Group III: pathophysiology. Circulation. 2004; 109: 2617-25.

40. Dandona P, Aljada A, Bandyopadhyay A. Inflammation: the link between insulin resistance, obesity and diabetes. Trends Immunol. 2004; 25: 4-7.

41. Han Y, Qiu H, Pei X, Fan Y, Tian H, Geng J. Low-dose Sinapic Acid Abates the Pyroptosis of Macrophages by Downregulation of IncRNA-MALAT1 in Rats With Diabetic Atherosclerosis. J Cardiovasc Pharmacol. 2018; 71: 104-12.

42. Falcao-Pires I, Leite-Moreira AF. Diabetic cardiomyopathy: understanding the molecular and cellular basis to progress in diagnosis and treatment. Heart Fail Rev. 2012; 17: 325-44.

43. Jia G, Whaley-Connell A, Sowers JR. Diabetic cardiomyopathy: a hyperglycaemia- and insulin-resistance-induced heart disease. Diabetologia. 2018; 61: 21-8 
44. Su XF, Sun L, Liu BL, Tao XJ, Li HQ, Li FF, et al. An Intensive Diabetes Screening and Treatment Program Improves Diagnosis, Treatment and Outcomes of Diabetes in Patients Admitted with Cardiac Diseases. Exp Clin Endocrinol Diabetes. 2017; 125: 151-5.

45. Li G, Zou L, Xie W, Wen S, Xie Q, Gao Y, et al. The effects of NONRATT021972 IncRNA siRNA on PC12 neuronal injury mediated by P2X7 receptor after exposure to oxygen-glucose deprivation. Purinergic signalling. 2016; 12: 479-87.

46. Zhang M, Gu H, Xu W, Zhou X. Down-regulation of IncRNA MALAT1 reduces cardiomyocyte apoptosis and improves left ventricular function in diabetic rats. Int J Cardiol. 2016; 203: 214-6.

47. Bacci L, Barbati SA, Colussi C, Aiello A, Isidori AM, Grassi C, et al. Sildenafil normalizes MALAT1 level in diabetic cardiomyopathy. Endocrine. 2018.

48. Starley BQ, Calcagno CJ, Harrison SA. Nonalcoholic fatty liver disease and hepatocellular carcinoma: a weighty connection. Hepatology. 2010; 51: 1820-32.

49. Matteoni CA, Younossi ZM, Gramlich T, Boparai N, Liu YC, McCullough AJ. Nonalcoholic fatty liver disease: a spectrum of clinical and pathological severity. Gastroenterology. 1999; 116: 1413-9.

50. Rafiq N, Bai C, Fang Y, Srishord M, McCullough A, Gramlich T, et al. Long-term follow-up of patients with nonalcoholic fatty liver. Clin Gastroenterol Hepatol. 2009; 7: 234-8.

51. Wong RJ, Cheung R, Ahmed A. Nonalcoholic steatohepatitis is the most rapidly growing indication for liver transplantation in patients with hepatocellular carcinoma in the U.S. Hepatology. 2014; 59: 2188-95.

52. Li C, Chen J, Zhang K, Feng B, Wang R, Chen L. Progress and Prospects of Long Noncoding RNAs (IncRNAs) in Hepatocellular Carcinoma. Cell Physiol Biochem. 2015; 36: 423-34.

53. Leti F, Legendre C, Still CD, Chu X, Petrick A, Gerhard GS, et al. Altered expression of MALAT1 IncRNA in nonalcoholic steatohepatitis fibrosis regulates CXCL5 in hepatic stellate cells. Transl Res. 2017; 190: 25-39 e21.

54. Farrell GC, Larter CZ. Nonalcoholic fatty liver disease: from steatosis to cirrhosis. Hepatology. 2006; 43: S99-S112.

55. Gibson RS, Gibson IL, Webber CE, Atkinson SA. An improved multi-element measurement of mineral absorption in the piglet utilizing the fecal monitoring technique. Biol Trace Elem Res. 1988; 17: 139-49.

56. Paradis V, Perlemuter G, Bonvoust F, Dargere D, Parfait B, Vidaud M, et al. High glucose and hyperinsulinemia stimulate connective tissue growth factor expression: a potential mechanism involved in progression to fibrosis in nonalcoholic steatohepatitis. Hepatology. 2001; 34: 738-44.

57. Ali T, Hasan M, Hamadani M, Harty RF. Gastroparesis. South Med J. 2007; 100: 281-6.

58. Goyal RK, Spiro HM. Gastrointestinal manifestations of diabetes mellitus. Med Clin North Am. 1971; 55: 1031-44.

59. Verne GN, Sninsky CA. Diabetes and the gastrointestinal tract. Gastroenterol Clin North Am. 1998; 27: 861-74, vi-vii.

60. De Block CE, De Leeuw IH, Pelckmans PA, Van Gaal LF. Current concepts in gastric motility in diabetes mellitus. Curr Diabetes Rev. 2006; 2: 113-30.

61. Horvath VJ, Vittal H, Lorincz A, Chen H, Almeida-Porada G, Redelman D, et al. Reduced stem cell factor links smooth myopathy and loss of interstitial cells of cajal in murine diabetic gastroparesis. Gastroenterology. 2006; 130: 759-70.

62. Bichu P, Nistala R, Khan A, Sowers JR, Whaley-Connell A. Angiotensin receptor blockers for the reduction of proteinuria in diabetic patients with overt nephropathy: results from the AMADEO study. Vasc Health Risk Manag. 2009; 5: 129-40.

63. Afkarian M, Sachs MC, Kestenbaum B, Hirsch IB, Tuttle KR, Himmelfarb J, et al. Kidney disease and increased mortality risk in type 2 diabetes. J Am Soc Nephrol. 2013; 24: 302-8.

64. Kanwar YS, Sun L, Xie P, Liu FY, Chen S. A glimpse of various pathogenetic mechanisms of diabetic nephropathy. Annu Rev Pathol. 2011; 6: 395-423.

65. Hu M, Wang R, Li X, Fan M, Lin J, Zhen J, et al. LncRNA MALAT1 is dysregulated in diabetic nephropathy and involved in high glucose-induced podocyte injury via its interplay with beta-catenin. J Cell Mol Med. 2017; 21: 2732-47.

66. Li X, Zeng L, Cao C, Lu C, Lian W, Han J, et al. Long noncoding RNA MALAT1 regulates renal tubular epithelial pyroptosis by modulated miR-23c targeting of ELAVL1 in diabetic nephropathy. Exp Cell Res. 2017; 350: 327-35.

67. Wu D, Cheng YG, Huang X, Zhong MW, Liu SZ, Hu SY. Downregulation of IncRNA MALAT1 contributes to renal functional improvement after duodenal-jejunal bypass in a diabetic rat model. J Physiol Biochem. 2018; 74: 431-9.

68. Metzger BE, Coustan DR. Summary and recommendations of the Fourth International Workshop-Conference on Gestational Diabetes Mellitus. The Organizing Committee. Diabetes Care. 1998; 21 Suppl 2: B161-7.

69. Buchanan TA, Xiang A, Kjos SL, Watanabe R. What is gestational diabetes? Diabetes Care. 2007; 30 Suppl 2: S105-11.

70. Retnakaran R, Ye C, Kramer CK, Hanley AJ, Connelly PW, Sermer M, et al. Impact of daily incremental change in environmental temperature on beta cell function and the risk of gestational diabetes in pregnant women. Diabetologia. 2018.

71. Zhang Y, Wu H, Wang F, Ye M, Zhu H, Bu S. Long non-coding RNA MALAT1 expression in patients with gestational diabetes mellitus. Int J Gynaecol Obstet. 2018; 140: 164-9. 